\title{
Cascaded emission of linearly polarized single photons from positioned InP/GalnP quantum dots
}

Cite as: Appl. Phys. Lett. 103, 191113 (2013); https://doi.org/10.1063/1.4828354

Submitted: 16 July 2013 • Accepted: 16 October 2013 • Published Online: 07 November 2013

T. Braun, S. Unsleber, V. Baumann, et al.
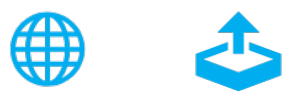

View Online

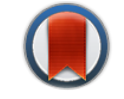

CrossMark

\section{ARTICLES YOU MAY BE INTERESTED IN}

Enhanced single photon emission from positioned InP/GalnP quantum dots coupled to a confined Tamm-plasmon mode

Applied Physics Letters 106, 041113 (2015); https://doi.org/10.1063/1.4907003

Electrically driven quantum dot-micropillar single photon source with $34 \%$ overall efficiency

Applied Physics Letters 96, 011107 (2010); https://doi.org/10.1063/1.3284514

Single-photon emission at $1.5 \mu \mathrm{m}$ from an InAs/InP quantum dot with highly suppressed multiphoton emission probabilities

Applied Physics Letters 109, 132106 (2016); https://doi.org/10.1063/1.4961888

母QBLOX

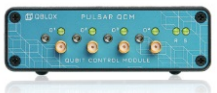

1 qubit
Shorten Setup Time Auto-Calibration More Qubits

Fully-integrated Quantum Control Stacks Ultrastable DC to $18.5 \mathrm{GHz}$ Synchronized $<<1$ ns Ultralow noise

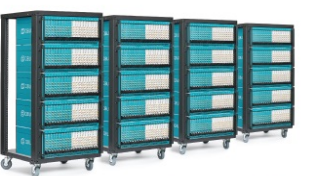

100s qubits

visit our website > 


\title{
Cascaded emission of linearly polarized single photons from positioned InP/GalnP quantum dots
}

\author{
T. Braun, ${ }^{1}$ S. Unsleber, ${ }^{1}$ V. Baumann, ${ }^{1}$ M. Gschrey, ${ }^{2}$ S. Rodt, ${ }^{2}$ S. Reitzenstein, ${ }^{2}$ \\ C. Schneider, ${ }^{1}$ S. Höfling, ${ }^{1}$ and M. Kamp ${ }^{1}$ \\ ${ }^{1}$ Technische Physik, Physikalisches Institut and Wilhelm Conrad Röntgen-Research Center for Complex \\ Material Systems, Universität Würzburg, Am Hubland, D-97074 Würzburg, Germany \\ ${ }^{2}$ Institut für Festkörperphysik, Technische Universität Berlin, Hardenbergstrasse 36, 10623 Berlin, Germany
}

(Received 16 July 2013; accepted 16 October 2013; published online 7 November 2013)

\begin{abstract}
We report on the optical characterization of site-controlled InP/GaInP quantum dots (QDs). Spatially resolved low temperature cathodoluminescence proves the long-range ordering of the buried emitters, revealing a yield of $\sim 90 \%$ of optically active, positioned QDs and a strong suppression of emitters on interstitial positions. The emission of single QDs shows a pronounced degree of linear polarization along the $[0,-1,1]$ crystal axis with an average degree of polarization of 94\%. Photon correlation measurements of the emission from a single QD indicate the singlephoton character of the exciton and biexciton emission lines as well as the cascaded nature of the photon pair. (C) 2013 AIP Publishing LLC. [http://dx.doi.org/10.1063/1.4828354]
\end{abstract}

The groundbreaking demonstration of single photon emission from semiconductor quantum dots ${ }^{1}$ has triggered an extensive amount of research devoted to their nonclassical emission character. Furthermore, the integration of quantum dots (QDs) into tailored photonic environments such as micro- and nanocavities has allowed the observation of pronounced coupling effects between light and matter. ${ }^{2,3}$ The enhancement of the spontaneous emission rate experienced by a single QD integrated in a microcavity has already been exploited to increase the efficiency of optically ${ }^{4,5}$ and electrically driven single photon sources, ${ }^{6}$ and of sources of polarization entangled photons. ${ }^{7}$ To fabricate such devices with high yield, only a single QD should be located in the active region, specifically at the position of the highest optical field intensity. This is essential in order to suppress the emission of uncorrelated photons from spectator QDs in the device, and to achieve maximum light-matter coupling. To date, however, most devices have been realized based on randomly grown QDs, which implies that the aforementioned criterion can only be fulfilled with low yield. QD positioning techniques allow one to control the spatial matching between emitter and cavity mode, as has been reported elsewhere. ${ }^{8,9}$ For some applications, such as quantum key distribution using single photons, the overall efficiency of the system can be increased by using quantum light sources which emit photons of a specified polarization. Consequently, work has been devoted to the development of polarization controlled quantum dot based single photon sources by post-processing techniques of grown cavities, ${ }^{10,11}$ since conventional InAs/GaAs QDs emit single photons with unspecified polarization. In contrast, the emission from our InP/GaInP site-controlled QDs (SCDQs) is characterized by a very high degree of linear polarization.

Furthermore, the emission wavelength of the QDs should be in the high-sensitivity region of commercial single photon detection modules. This is, unfortunately, not the case in the well-established InAs/GaAs material system. However, InP/GaInP QDs, grown on GaAs substrates can indeed emit single photons on demand in the visible red spectral range where silicon single photon detectors have their highest quantum efficiency. ${ }^{12}$ Hence, site-controlled InP/GaInP QDs with high optical quality are promising candidates to meet the aforementioned requirements, and first promising experimental demonstrations of such nanostructures have recently been reported. ${ }^{13,14}$

So far, mainly the emission properties of self assembled InP/GaInP quantum dots were investigated. Reischle et al. studied photon correlations between the biexciton and the exciton emission and analyzed the influence of the dark exciton state on the optical and quantum optical properties. ${ }^{15}$ Polarization properties of single InP QDs were investigated by Sugisaki et al. ${ }^{16}$ and Schmidbauer et al. ${ }^{17}$ Both groups reported a surprisingly high degree of linear polarization from the QDs, which they attributed to anisotropic strain in the matrix and composition modulations ${ }^{15}$ or a shape anisotropy of the QDs. ${ }^{16}$ Zwiller et al. initially demonstrated the capability of InP QDs to act as sources of triggered single photons, ${ }^{12}$ which also has been confirmed by Baumann et al. for site-controlled InP QDs. ${ }^{13}$

In this letter, we extend the work by Baumann et al. ${ }^{13,14}$ and focus on the emission properties of site-controlled InP QDs with respect to their exploitation as the active medium in quantum light sources in greater detail. We present a detailed analysis on the polarization characteristics and the cascaded nature of the emission of the exciton and biexciton of those QDs, which has been elusive to date. The sample under investigation was realized by means of epitaxial growth on a pre-patterned substrate with shallow nanoholes via gas-source molecular beam epitaxy. Details on the fabrication process can be found elsewhere. ${ }^{13,14}$ The optically active QDs are vertically separated from the etched nanoholes by approximately $40 \mathrm{~nm}$, which is facilitated by the introduction of a strain mediating seeding layer. Such a large separation ensures the preservation of high optical properties of the site-controlled QDs. ${ }^{9}$

In order to assess the spatial ordering of our buried QDs under investigation, we characterize the sample via spatially resolved scanning cathodoluminescence (CL) spectroscopy. 
This technique has been proven to be very suitable for the investigation of long-range ordering effects of optically active emitters, since it combines a high spatial resolution with the capability of probing the emitter's luminescence properties. ${ }^{18-20}$ The corresponding panchromatic CL image of a QD array with a spacing of $1.25 \mu \mathrm{m}$, recorded at a temperature of $5 \mathrm{~K}$ is shown in Fig. 1. The CL intensity map was measured with an acceleration voltage of $7 \mathrm{kV}$ and a beam current of $0.8 \mathrm{nA}$. Spectra were taken every $100 \mathrm{~nm}$, and the resulting image shows excellent long-range ordering and more than $90 \%$ of optically active QDs. The strong variation in the brightness (from 3\% to $100 \%$ ) of the QDs can be attributed to a variation in the quantum efficiencies of our QDs, an effect which is of particular importance for sitecontrolled QDs. ${ }^{21}$ Less than 3\% of the QDs are misplaced, which indicates the high quality of the fabrication process.

Since $97 \%$ of the emitters are positioned QDs, their optical properties can be studied with micro-photoluminescence ( $\mu \mathrm{PL}$ ), which by itself cannot distinguish between on-site and misplaced dots on the sub-micron scale. The QDs were excited with a frequency doubled Nd:YAG-laser emitting at a wavelength of $532 \mathrm{~nm}$. The beam was focused through a $20 \times$ microscope objective to a $4 \mu \mathrm{m}$ spot on the sample which was held at a temperature of $18 \mathrm{~K}$. The photoluminescence signal was analyzed by a monochromator equipped with a 1200 lines/mm grating and a liquid nitrogen cooled Silicon-CCD, yielding an overall spectral resolution of about $30 \mu \mathrm{eV}$ at an energy of $1.88 \mathrm{eV}$. Figure 2(a) depicts a typical $\mathrm{QD} \mu \mathrm{PL}$ spectrum recorded at an excitation power of $4.7 \mu \mathrm{W}$ in horizontal and vertical polarization, respectively. Two main emission features dominate the spectrum, which we attribute to the exciton (X) and biexciton (XX) emission of a single QD with a linewidth of $281 \mu \mathrm{eV}$ and $270 \mu \mathrm{eV}$, respectively.

Figure 2(b) shows the power dependent integrated intensity of both emission lines. The exciton emission shows an almost linear behaviour with a slope of 0.91 , whereas the biexciton line has a slope of 1.59 which is in agreement with previous studies, ${ }^{13}$ however it deviates from the ideal coefficient of 2. This deviation can possibly be explained by the presence of non-radiative relaxation channels, such as defects in the vicinity of the QD, which are induced by the

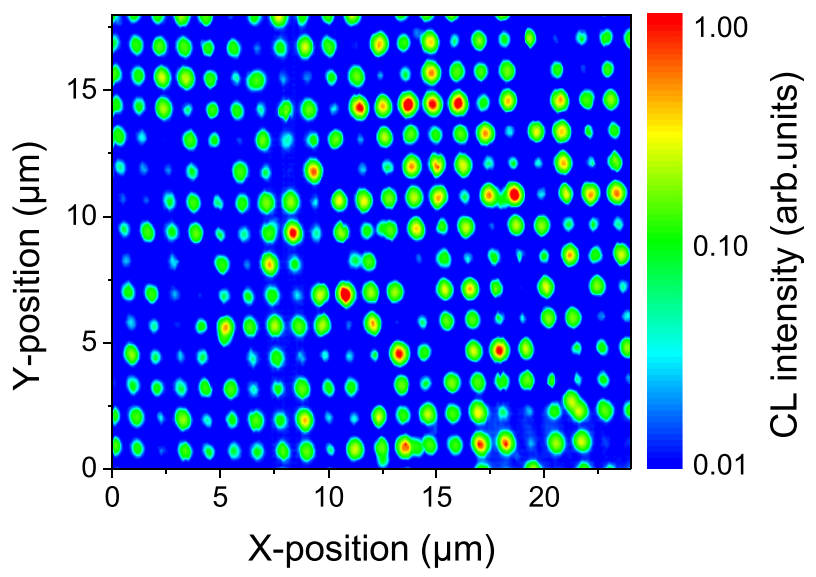

FIG. 1. (a) Panchromatic CL intensity image of site-controlled QDs with a pitch of $1.25 \mu \mathrm{m}$, revealing the excellent long-range ordering and high yield of optically active emitters. (a)
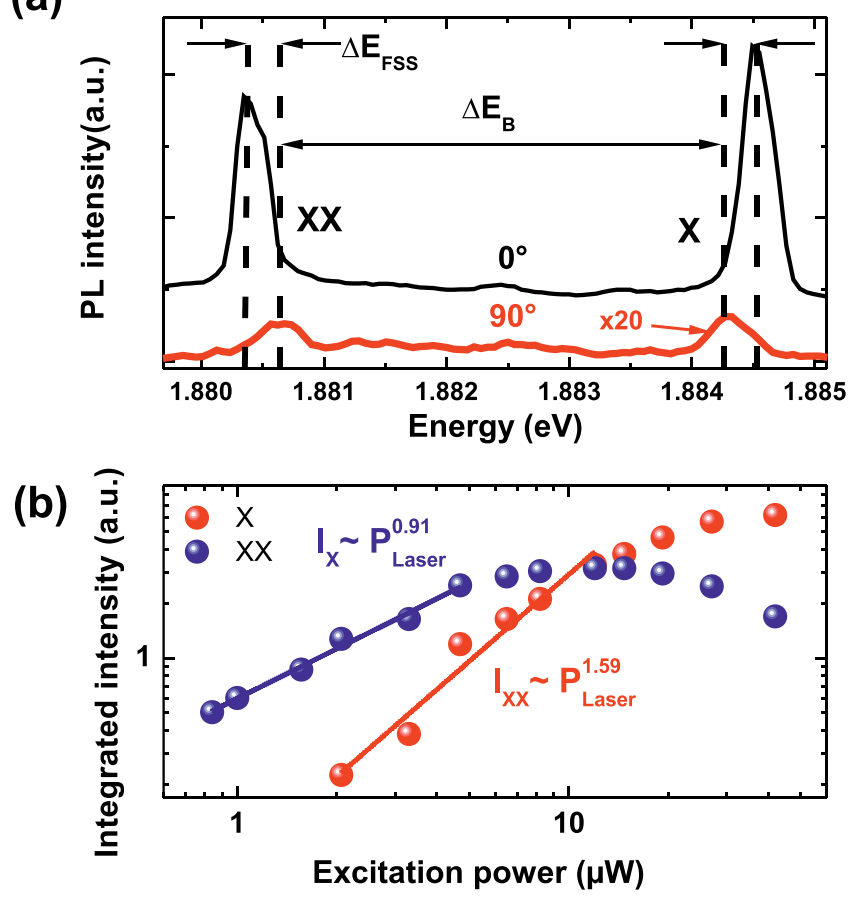

FIG. 2. (a) $\mu \mathrm{PL}$ spectrum of a single $\mathrm{X}-\mathrm{XX}$ pair at two different angles of the polarizer showing $\mathrm{H}$ and $\mathrm{V}$ polarization. (b) Integrated intensities of the emission features as a function of the excitation power.

pre-patterning process. However, due to the deviation from the ideal power-law coefficients, the assignment of exciton and biexciton is not completely free of ambiguities. Interestingly, no clear spectral signatures of charged exciton complexes were found for the investigated QDs.

To further investigate the optical properties of the QDs, we have performed polarization dependent measurements: A linear polarizer in combination with a quarter-waveplate was used to select a specific polarization. The resulting QD spectrum at arbitrary polarizer position averages the two contributions with the polarization angle, and the peak energy oscillates between the extreme values of purely $\mathrm{H}$ and $\mathrm{V}$ polarized light (which represents the fine structure splitting). Fig. 2(a) depicts two selected, polarization resolved spectra where for a polarizer angle of $0^{\circ}$ only the $\mathrm{H}$-mode and for $90^{\circ}$ the $\mathrm{V}$-mode is detectable, respectively. The dashed lines indicate the extracted fine structure splitting of $\sim 235 \mu \mathrm{eV}$.

Fig. 3(a) depicts the integrated intensity of the QD emission shown in Fig. 2(a) over the position of the linear polarizer. Both the exciton and the biexciton exhibit a surprisingly pronounced linear polarization, with the orthogonal polarization being suppressed by more than two orders of magnitude. The degree of polarization of the exciton is $P_{X}=\frac{I_{\max }-I_{\min }}{I_{\max }+I_{\min }}$ $=0.982$ and $P_{X X}=0.985$ for the biexciton for this particular $\mathrm{QD}$, and as large as 0.94 on average on our sample, which is much larger than the observed values for self-assembled $\mathrm{QDs}^{16,18}$ in this material system. We anticipate that the observed strong optical anisotropy arises from the anisotropy in the microscopic structure of the SCQDs, similar to reports on self-assembled InP QDs. ${ }^{16}$ The $\mathrm{Ga}_{0.51} \mathrm{In}_{0.49} \mathrm{P}$-matrix also shows a high degree of polarization which is attributed to a lateral composition modulation of Ga and In. Similar crystal ordering effects, enhanced, e.g., by an imperfect 

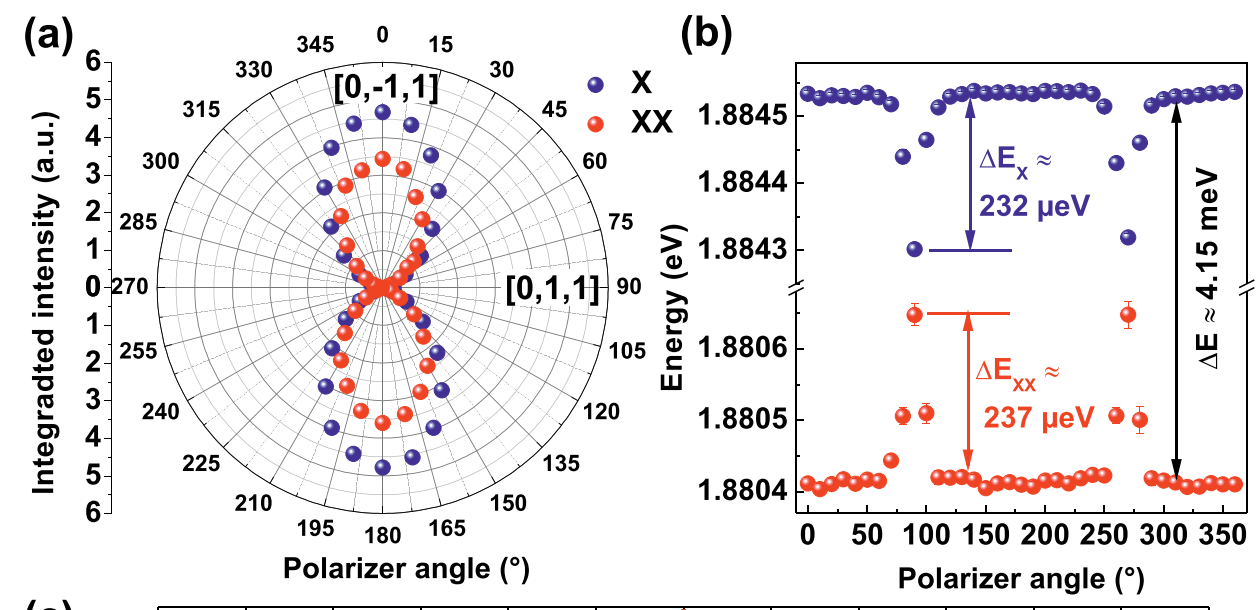

(c)

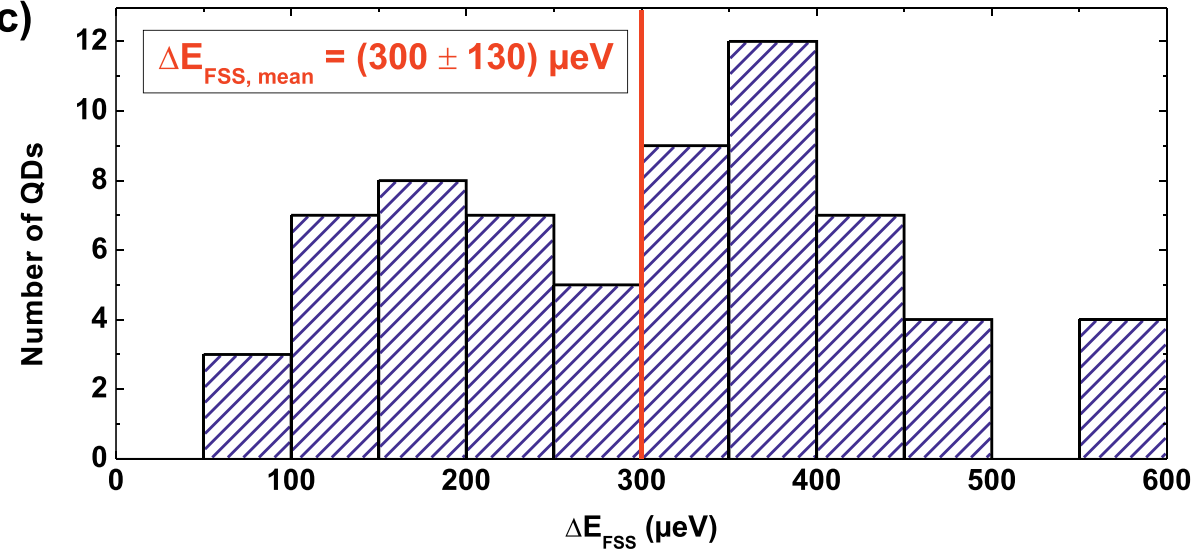

FIG. 3. (a) Polarization graph of the X-XX emission pair in Fig. 2. (b) Peak energy of the emission lines as a function of the polarization angle to extract the fine structure splitting. (c) Statistical histogram of the fine structure splitting of $\sim 66$ QDs. lattice matching to GaAs, could cause this peculiar behavior. The polarization dependent measurements also allow for investigations of the fine structure splitting between both polarization axes.

Since the brighter mode, which is polarized along the $[0,-1,1]$ axis, dominates the $\mu \mathrm{PL}$-spectrum, the $[0,1,1]$ polarized mode can only be detected when the brighter mode is fully suppressed. This can clearly be seen when comparing the spectra taken at a polarizer angle of $0^{\circ}$ and $90^{\circ}$ in Fig. 2(a). Only for angles from $80^{\circ}$ to $100^{\circ}$ the contribution of the weaker mode is observable at all. This explains the peculiar behavior in Fig. 3(b) where the fitted emission energies for the two QD-emission lines are plotted with respect to the angle of the linear polarizer. Both curves are opposite in phase and thus strengthen our assignment of exciton and biexciton emissions from the same QD. The evaluation of the curve results in a fine structure splitting (FSS) of the exciton $\Delta E_{X}=(232 \pm 5) \mu \mathrm{eV}$ and the biexciton $\Delta E_{X X}=(237 \pm 5) \mu \mathrm{eV}$, which gives in total $\Delta E_{F S S}=(234.5 \pm 3.5) \mu \mathrm{eV}$. The biexciton binding energy amounts to $\Delta E=4.15 \mathrm{meV}$, which is comparable to values measured for InAs/GaAs QDs. ${ }^{22}$

For a statistical analysis, polarization resolved $\mu \mathrm{PL}$ measurements were carried out on 66 QDs. All QDs under investigation exhibited the same direction of linear polarization along the $[0,-1,1]$ axis, which again indicates that the crystal structure itself causes this high degree of optical polarization. The extracted values for the fine structure splitting are shown in Fig. 3(c). The average extracted FSS amounts to $\Delta E_{F S S \text {,mean }}=300 \mu \mathrm{eV}$ and is comparable to splittings measured by Sugisaki et al. ${ }^{16}$ It is worth mentioning that the strong linear polarization of the QDs in combination with the QD's linewidth of 180-600 $\mu \mathrm{eV}$ set a limit the accurately extractable splitting. In fact, the error of the extracted value for the FSS significantly increases above $10 \%$ once the value of the splitting becomes smaller than $90 \mu \mathrm{eV}$, if we take the strongly polarized emission of our QD lines into account. We suggest that such large values for the FSS originate from a highly anisotropic QD shape, breaking the systems $\mathrm{D}_{2 \mathrm{D}}$ symmetry. Since structural investigations on uncapped QDs have revealed symmetric QD shapes, ${ }^{14}$ we suggest that a shape transition of the QD takes place during the capping procedure.

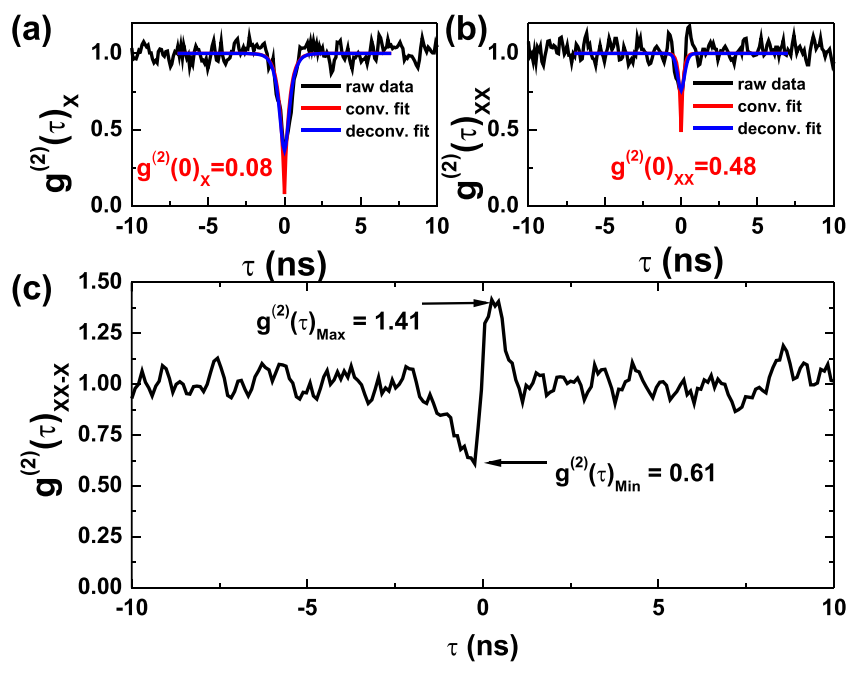

FIG. 4. (a) Photon auto-correlation measurement of the exciton, (b) the biexciton, and (c) cross-correlation of the X-XX emission. 
In order to assess the non-classical emission character of the photons from our QD, we performed photon autocorrelation measurements of both the excitonic and the biexcitonic emissions feature using a fiber coupled Hanbury Brown and Twiss configuration. The corresponding second order autocorrelation functions $\mathrm{g}^{(2)}(\tau)$ shown in Figs. 4(a) and 4(b) for the exciton and the biexciton reveal strong antibunching as expected from a non-classical light emitter. In particular, photon emission with a low probability of multi-photon events is reflected by the observed value of $\mathrm{g}^{(2)}(0)_{\mathrm{X}}$, deconv. fit $=0.08\left(\mathrm{~g}^{(2)}(0)_{\mathrm{X}}\right.$, conv. fit $\left.=0.37\right)$ for the exciton emission, when taking the temporal resolution of the setup of $\sim 400 \mathrm{ps}$ into account. Hereby $\mathrm{g}^{(2)}(0)_{\text {conv. fit }}$ corresponds to the $g^{(2)}(0)$-value extracted by fitting the data points with an exponential decay convoluted with a Gaussian detector response function (FWHM $400 \mathrm{ps}$ ). Due to a higher background affecting the biexciton emission, this emission line shows a slightly weaker suppression of multiphoton-events leading to a deconvoluted $\mathrm{g}^{(2)}(0)$-value of $\mathrm{g}^{(2)}(0)_{\mathrm{XX}}$, deconv. fit $=0.49\left(\mathrm{~g}^{(2)}(0)_{\mathrm{X}, \text { conv. fit }}=0.76\right)$.

Moreover, in order to give a clear evidence of the cascaded nature of the emission events, we performed crosscorrelation measurements on the exciton and the biexciton emissions. Two monochromators were used for a spectral filtering of the individual emission lines. The second-order cross-correlation function $\mathrm{g}^{(2)}(\tau)_{\mathrm{X}-\mathrm{XX}}$ is shown in Fig. 4(c) taken at a Power of $9.4 \mu \mathrm{W}$, i.e., when exciton and biexciton have comparable intensity (cf. Fig. 2(b)). Here the emission of the biexciton acts as the starting signal of the measurement, whereby the exciton emission triggers the stop avalance photodiode. The asymmetric anti-bunching (for $\tau<0$ ) and bunching $(\tau>0)$ behavior demonstrates the cascaded emission with a minimum value of $g_{X-X X}^{(2)} \approx 0.61$ and a maximum of $g_{X-X X}^{(2)} \approx 1.41$, respectively.

In conclusion, we have demonstrated the capability of site-controlled InP QDs to brightly emit red, single photons and cascaded photons from the exciton-biexciton cascade. The strong linear polarization of these QDs makes them very appealing for single photon applications in quantum communication systems. In order to exploit our QD system for the generation of polarization entangled photon pairs from the biexciton-exciton cascade, we suggest to apply further tuning techniques such as external strain and electric fields. ${ }^{23}$ Furthermore, we anticipate that similar techniques as deployed by Juska et al. ${ }^{24}$ can be applied to realize periodic arrays of red emitting QDs with vanishing fine structure splitting, taking advantage of substrate orientations of higher symmetries.
This work was financially supported by the German Ministry of Education and Research within the projects "QuaHL-Rep" and "QPENS." Technical assistance by T. Steinl during sample preparation and theoretical support by A. Schliwa is gratefully acknowledged.

${ }^{1}$ P. Michler, A. Kiraz, C. Becher, W. V. Schoenfeld, P. M. Petroff, L. Zhang, E. Hu, and A. Imamoglu, Science 290, 2282 (2000).

${ }^{2}$ J. Claudon, J. Bleuse, N. S. Malik, M. Bazin, P. Jaffrennou, N. Gregersen, C. Sauvan, P. Lalanne, and J. M. Gerard, Nat. Photonics 4, 174-177 (2010).

${ }^{3}$ J. P. Reithmaier, G. Sek, A. Löffler, C. Hofmann, S. Kuhn, S. Reitzenstein, L. V. Keldysh, V. D. Kulakovskii, T. L. Reinecke, and A. Forchel, Nature 432, 197-200 (2004).

${ }^{4}$ M. Pelton, C. Santori, J. Vučković, B. Zhang, G. S. Solomon, J. Plant, and Y. Yamamoto, Phys. Rev. Lett. 89, 233602 (2002).

${ }^{5}$ E. Moreau, I. Robert, L. Manin, V. Thierry-Mieg, J. M. Gérard, and I. Abram, Physica E (Amsterdam) 13, 418 (2002).

${ }^{6}$ T. Heindel, C. Schneider, M. Lermer, S. H. Kwon, T. Braun, S. Reitzenstein, S. Höfling, M. Kamp, and A. Forchel, Appl. Phys. Lett. 96, 011107 (2010).

${ }^{7}$ A. Dousse, J. Suffczynski, A. Beveratos, O. Krebs, A. Lemattre, I. Sagnes, J. Bloch, P. Voisin, and P. Senellart, Nature 466, 217 (2010).

${ }^{8}$ O. G. Schmidt, Lateral Alignment of Epitaxial Quantum Dots (Springer, Berlin, 2007).

${ }^{9}$ C. Schneider, A. Huggenberger, T. Sünner, T. Heindel, M. Strauß, S. Göpfert, P. Weinmann, S. Reitzenstein, L. Worschech, M. Kamp, S. Höfling, and A. Forchel, Nanotechnology 20, 434012 (2009).

${ }^{10}$ S. Strauf, N. G. Stoltz, M. T. Rakher, L. A. Coldren, P. M. Petroff, and D. Bouwmeester, Nat. Photonics 1, 704 (2007).

${ }^{11}$ M. T. Rakher, N. G. Stoltz, L. A. Coldren, P. M. Petroff, and D. Bouwmeester, Appl. Phys. Lett. 93, 091118 (2008).

${ }^{12}$ V. Zwiller, T. Aichele, W. Seifert, J. Persson, and O. Benson, Appl. Phys. Lett. 82, 1509 (2003).

${ }^{13}$ V. Baumann, F. Stumpf, C. Schneider, S. Kremling, L. Worschech, A. Forchel, S. Höfling, and M. Kamp, Appl. Phys. Lett. 100, 091109 (2012).

${ }^{14}$ V. Baumann, F. Stumpf, T. Steinl, A. Forchel, C. Schneider, S. Höfling, and M. Kamp, Nanotechnology 23, 375301 (2012).

${ }^{15}$ M. Reischle, G. J. Beirne, R. Roßbach, M. Jetter, and P. Michler, Phys. Rev. Lett. 101, 146402 (2008).

${ }^{16}$ M. Sugisaki, H. W. Ren, and S. V. Nair, Phys. Rev. B 59, R5300 (1999).

${ }^{17}$ M. Schmidbauer, F. Hatami, M. Hanke, P. Schäfer, K. Braune, W. T. Masselink, and R. Köhler, Phys. Rev. B 65, 125320 (2002).

${ }^{18}$ C. Schneider, A. Huggenberger, M. Gschrey, P. Gold, S. Rodt, A. Forchel, S. Reitzenstein, S. Höfling, and M. Kamp, Phys. Status Solidi A 209, 2379-2386 (2012).

${ }^{19}$ S. Rodt, A. Schliwa, K. Pötschke, F. Guffarth, and D. Bimberg, Phys. Rev. B 71, 155325 (2005).

${ }^{20}$ E. Pelucchi, S. Watanabe, K. Leifer, Q. Zhu, B. Dwir, P. De Los Rios, and E. Kapon, Nano Lett. 7, 1282-1285 (2007).

${ }^{21}$ F. Albert, S. Stobbe, C. Schneider, T. Heindel, S. Reitzenstein, S. Höfling, P. Lodahl, L. Worschech, and A. Forchel, Appl. Phys. Lett. 96, 151102 (2010).

${ }^{22}$ D. Sarkar, H. P. van der Meulen, J. M. Calleja, J. M. Becker, R. J. Haug, and K. Pierz, J. Appl. Phys. 100, 023109 (2006).

${ }^{23}$ R. Trotta, E. Zallo, C. Ortix, P. Atkinson, J. D. Plumhof, J. van den Brink, A. Rastelli, and O. G. Schmidt, Phys. Rev. Lett. 109, 147401 (2012).

${ }^{24}$ G. Juska, V. Dimastrodonato, L. O. Mereni, A. Gocalinska, and E. Pelucchi, Nat. Photonics 7, 527 (2013). 